\title{
Characteristic features of the SERA multigene family in the malaria parasite
}

\author{
Nobuko Arisue ${ }^{1 *}$, Nirianne M. Q. Palacpac ${ }^{2}$, Takahiro Tougan ${ }^{1}$ and Toshihiro Horii ${ }^{2}$
}

\begin{abstract}
Serine repeat antigen (SERA) is conserved among species of the genus Plasmodium. Sera genes form a multigene family and are generally tandemly clustered on a single chromosome. Although all Plasmodium species encode multiple sera genes, the number varies between species. Among species, the members share similar sequences and gene organization. SERA possess a central papain-like cysteine protease domain, however, in some members, the active site cysteine residue is substituted with a serine. Recent studies implicate this gene family in a number of aspects in parasite biology and induction of protective immune response. This review summarizes the current understanding on this important gene family in several Plasmodium species. The Plasmodium falciparum (Pf)-sera family, for example, consists of nine gene members. Unlike other multigene families in Plasmodium species, Pf-sera genes do not exhibit antigenic variation. Pf-sera5 nucleotide diversity is also low. Moreover, although Pf-sera5 is highly transcribed during the blood stage of malaria infection, and a large amount is released into the host blood following schizont rupture, in malaria endemic countries the sero-positive rates for Pf-SERA5 are low, likely due to Pf-SERA5 binding of host proteins to avoid immune recognition. As an antigen, the N-terminal $47 \mathrm{kDa}$ domain of Pf-SERA5 is a promising vaccine candidate currently undergoing clinical trials. Pf-SERA5 and Pf-SERA6, as well as P. berghei (Pb)-SERA3, and Pb-SERA5, have been investigated for their roles in parasite egress. Two $P$. yoelii SERA, which have a serine residue at the protease active center, are implicated in parasite virulence. Overall, these studies provide insight that during the evolution of the Plasmodium parasite, the sera gene family members have increased by gene duplication, and acquired various functions that enable the parasite to survive and successfully maintain infection in the host.
\end{abstract}

Keywords: Plasmodium, SERA, Gene family, Function, Polymorphism

\section{Background}

Malaria, transmitted by the bite of mosquitoes infected with the Plasmodium parasite, is a life-threatening infectious disease. In 2018, there were an estimated 228 million cases and 405,000 related deaths [1], most of which were children in the WHO African region. After an unprecedented decrease in the malaria burden following introduction of $\mathrm{Coartem}^{\circledR}$, the decrease in malaria incidence and death has since reached a plateau, largely due to infrequent and unsustainable supply of current

\footnotetext{
*Correspondence: arisue@biken.osaka-u.ac.jp

${ }^{1}$ Research Center for Infectious Disease Control, Research Institute for Microbial Diseases, Osaka University, Suita, Osaka 565-0871, Japan

Full list of author information is available at the end of the article
}

interventions. An effective malaria vaccine is urgently needed, but its development is extremely challenging due to several immune evasion mechanisms both in the mosquito vector and human host.

Plasmodium falciparum serine repeat antigen (PfSERA) is an asexual blood stage antigen, so named because of the stretch of serine residues found in its amino acid sequence [2]. Pf-sera was initially considered a single gene; however, the $P$. falciparum genome project revealed that Pf-sera resides in a multi-locus forming gene family. Eight genes, Pf-sera1 to Pf-sera8, are clustered in tandem on chromosome 2 [3]. Pf-sera 9 was later found on chromosome 9 [4]. Notably, due to the $\mathrm{N}$-terminal truncated form of Pf-SERA8, it was previously considered a pseudogene; however, further studies

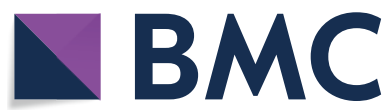

(c) The Author(s) 2020. This article is licensed under a Creative Commons Attribution 4.0 International License, which permits use, sharing, adaptation, distribution and reproduction in any medium or format, as long as you give appropriate credit to the original author(s) and the source, provide a link to the Creative Commons licence, and indicate if changes were made. The images or other third party material in this article are included in the article's Creative Commons licence, unless indicated otherwise in a credit line to the material. If material is not included in the article's Creative Commons licence and your intended use is not permitted by statutory regulation or exceeds the permitted use, you will need to obtain permission directly from the copyright holder. To view a copy of this licence, visit http://creativecommons.org/licenses/by/4.0/. The Creative Commons Public Domain Dedication waiver (http//creativecommons.org/publicdomain/zero/1.0/) applies to the data made available in this article, unless otherwise stated in a credit line to the data. 
demonstrated that $P f$-sera 8 was transcribed at the sporozoite stage [5]. The first identified Pf-SERA was Pf-sera5, which is the only family member containing a serine stretch. Its $\mathrm{N}$-terminal $47 \mathrm{kDa}$ domain (SE47) has been studied as a candidate vaccine antigen [6] and shows promise as (i) antibodies against this domain inhibit parasite growth in vitro [7-12]; and (ii) sero-epidemiological studies in malaria endemic areas have reported a negative correlation between parasitemia and anti-SE47 antibody titers $[13,14]$. When originally designated as a multigene family, SERA were believed to be important for antigenic variation, which refers to the parasite's ability to present a variety of antigenic molecules on the surface of infected red blood cells (RBC) to facilitate immune evasion [15]. However, the Pf-sera family, has been shown to not exhibit antigenic variation. Moreover, transcriptional analysis revealed that all $P f$-sera genes, except for $P f$ sera 8 , are transcribed nearly simultaneously [11].

All existing Plasmodium species have a sera multigene family; however, the number of genes varies between species $[16,17]$. Gene duplication and gene loss have repeatedly occurred during the parasite's evolution, allowing the parasite to undoubtedly develop new mechanisms for survival and maintenance of the delicate biological equilibrium of parasite and host. Following release of the $P$. falciparum draft genome, genome projects focused on several Plasmodium species have been accelerated resulting in characterization of the SERA multigene family structure in these species. In addition, functional studies have been performed at various points in the parasite life-cycle to better understand this multigene family. Herein, we review recent progress in these areas.

\section{Organization and evolutionary relationships of sera genes in $\mathbf{2 6}$ Plasmodium species}

The DNA sequence of $P$. falciparum chromosome 2 [3] provided the first insight into the sera multigene family. Eight genes, Pf-seral to Pf-sera 8 , were clustered in tandem between two conserved genes: a hypothetical protein gene and the iron-sulfur assembly protein gene. Currently, the Plasmodium genome data, available in public databases, has more than 200 sera gene sequences from 26 Plasmodium species (Fig. 1, Additional file 1: Table S1). Each Plasmodium has multiple numbers of sera genes that are generally tandemly arranged on a chromosome in similar order as $P f$-sera 1 to $P f$-sera 8 . Sera genes found outside of the cluster, such as $P f$-sera9, were found in avian malaria parasites and in Laverania, a subgenus of Plasmodium, which contain P. falciparum and related ape parasites. Among Laverania species, gene synteny was observed, with the location and arrangement of sera genes determined to be identical to that of Pf-sera, with the exception of $P$. reichenowi (chimpanzee parasite)

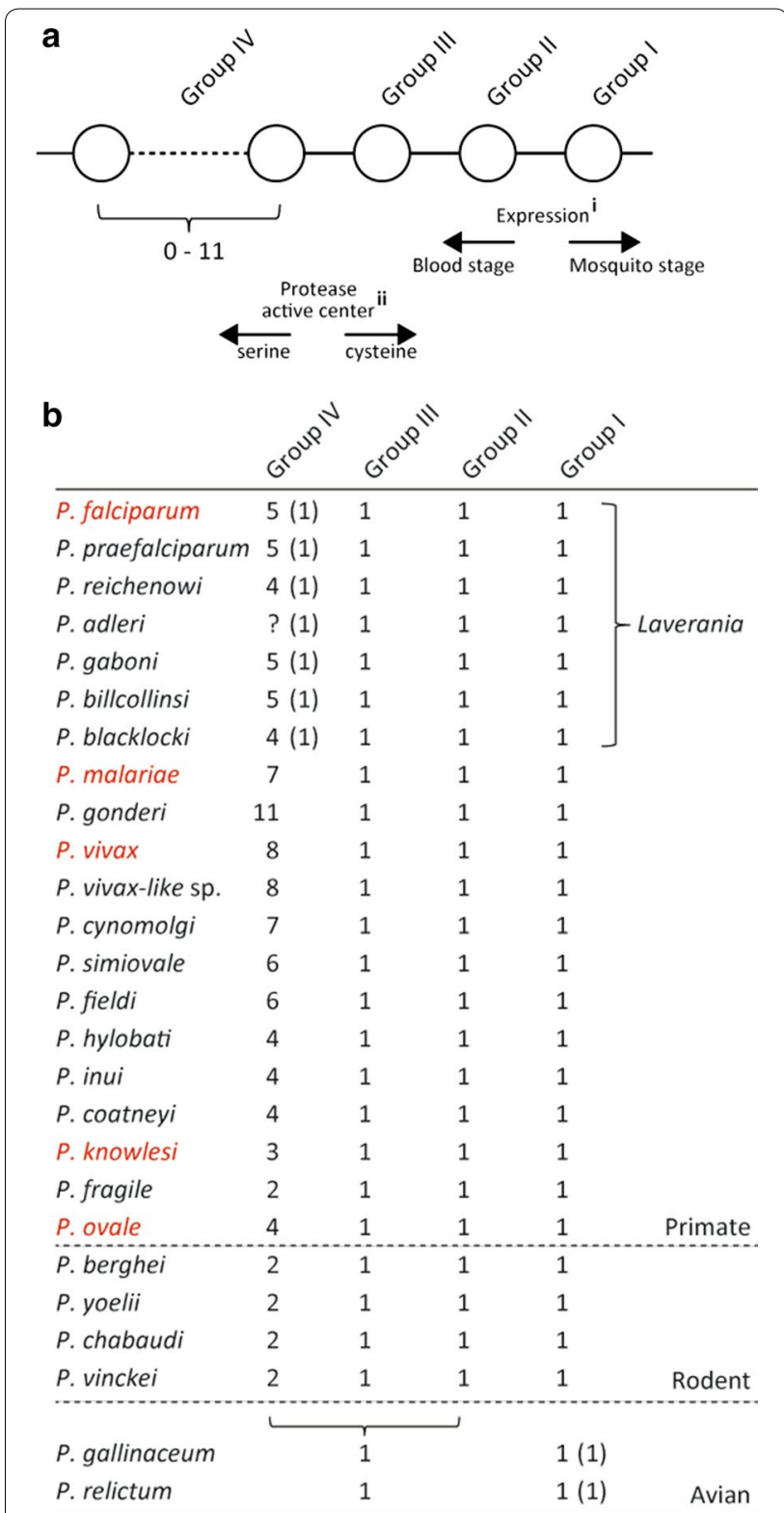

Fig. 1 Plasmodium sera gene family. a The organization of Plasmodium sera genes and their characteristic gene and protein features. Plasmodium sera genes (denoted as circles) are clustered in tandem and are categorized into four groups based on genetic background. In terms of protein expression, i: Group I SERA is expressed in the mosquito stage and Group II to IV are expressed in the host blood stage. In terms of the protease motif, ii: SERA members containing cysteine residues in the protease active center belong to Groups I to III, while those with a substituted serine residue belong to Group IV. The number of Group IV SERA genes can vary from 1 to 11. $\mathbf{b}$ The number of sera genes identified in each group. For Group IV sera and two Group I sera, the number of genes not in tandem cluster is shown in parenthesis. Plasmodium species with humans as a natural host are shown in red. The number of Group IV sera genes in $P$. adleri is currently unresolved 
and P. blacklocki (gorilla parasite), both of which lack the Pf-sera3 orthologue [18]. Of note, the number of sera genes in P. adleri (another gorilla parasite) also remains uncertain due to ambiguous genome mapping of the sera gene region [18]. Furthermore, in the avian $P$. relictum (Pr), Pr-sera1 and Pr-sera 2 are located in tandem on chromosome 4 while Pr-sera 3 is located on chromosome 9; meanwhile in the avian $P$. gallinaceum $(P g)$, the location of each gene has not yet been assigned to specific chromosomes. Nevertheless, Pg-sera1 and Pg-sera 2 have been identified as being tandemly located on the same contig while Pg-sera 3 is located elsewhere.

All of the SERA members contain a papain-like cysteine protease motif and can be classified into two major types based on the active site residue: a cysteinetype or serine-type SERA. Based on sequence similarity, sera genes can be categorized into four groups (Group I to IV) $[16,17]$ (Fig. 1). Group I to III contain cysteinetype SERA and Group IV contains a serine-type SERA. The sera gene IDs and their groupings are summarized in Additional file 1: Table S1. The maximum likelihood (ML) tree showed that each of the sera gene groups form monophyletic groups (Fig. 2a).

Transcription of Group I sera genes was detected primarily during the mosquito stage, while that of Group II to IV was observed during the blood stage in the vertebrate host [18]. Among 26 Plasmodium species, the number of sera genes in tandem cluster varied from 2 (in two avian parasites) to 14 (in $P$. gonderi, excluding a truncated sera gene [19]). Apart from avian parasites, the difference in the number of genes is always found in serine-type Group IV sera genes. The ML tree suggests that the generation of Group II and III sera genes would have occurred after the divergence of avian parasites, as sera1 of the avian parasites is positioned at the branch leading to the common ancestors of Group II and III sera genes [16]. The ML tree also suggested that gene duplication(s) of the Group IV sera genes occurred independently in each lineage, generating multiple numbers of Group IV sera genes. The number of Group IV sera genes remarkably increased in some primate Plasmodium species (Figs. 1b, 2b). Like other multigene families, the Plasmodium sera multigene family was considered to be driven by birth-and-death evolution [17]. This model assumes that new genes are created by gene duplications, and that some duplicated genes can be maintained in the genome for a long time while other genes become deleted or nonfunctional through deleterious mutations [20]. Gene loss, gene truncation, and pseudogenization were observed in Group IV sera genes of primate parasites except for P. malariae, P. ovale and Laveranian species [17]. These processes may be associated with an evolutionary event that involves the acquisition of a new enzymatic pathway,

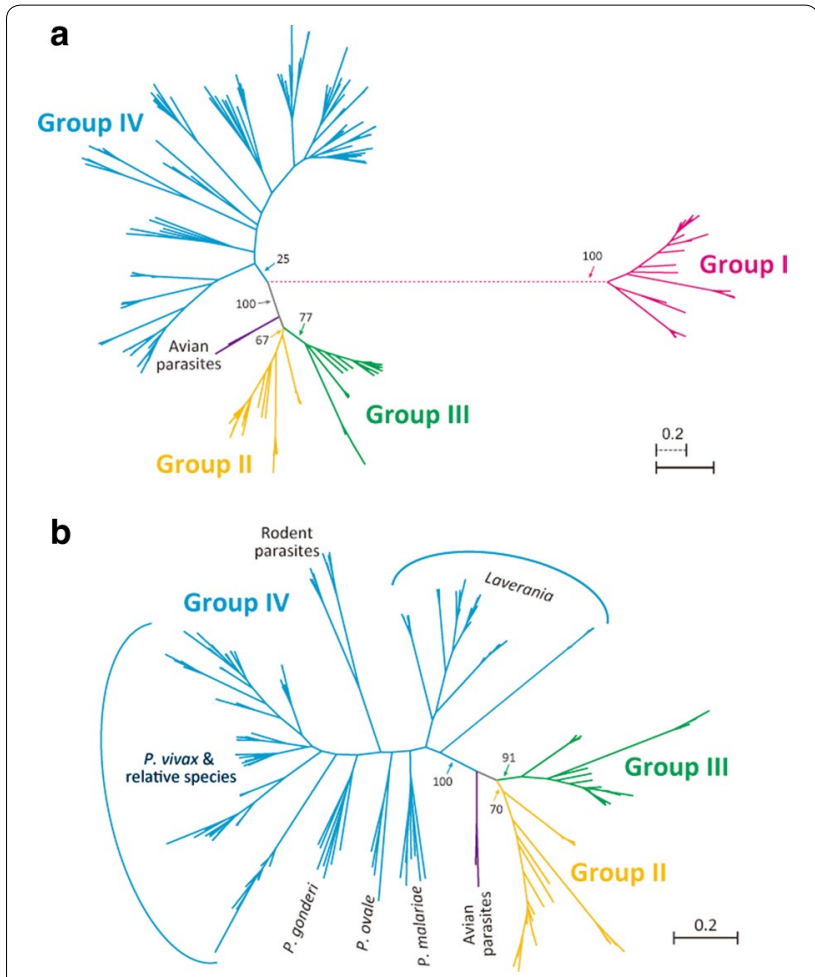

Fig. 2 Phylogenetic tree of sera genes inferred by the maximum likelihood (ML) method. The sequences of Plasmodium sera genes were translated to amino acid sequences and aligned by using MAFFT version 7.409 [58]. Site selection for tree inference was done with the Gblocks option, with a more stringent selection criteria implemented in SeaView version 4.7 [59]. Trees were inferred under the JTT $+\Gamma+$ I model. MEGA X [60] was used for the analysis. Bootstrap proportions from the ML methods with 100 replications are shown. a 300 amino acid positions from 195 Group I to IV sera genes were used for tree inference. The tree with the highest log likelihood $(-l i=28137.0426)$ is shown. A discrete $\Gamma$ distribution was used to model evolutionary rate differences among sites (4 categories, $+\Gamma$, parameter $=1.1871)$. The rate variation model allowed for some sites to be evolutionarily invariable (+1, 9.2557\% sites). b 424 amino acid positions from 173 Group II to IV sera genes were used for tree inference. The log likelihood of the tree is - 37969.9. A discrete $\Gamma$ distribution was used to model evolutionary rate differences among sites ( 4 categories, $+\Gamma$, parameter $=1.2356$ ). The rate variation model allowed for some sites to be evolutionarily invariable $(+1,5.0708 \%$ sites)

expansion of host range and/or activation of new roles in host immune evasion.

\section{Functions of SERA}

The gene structure and primary protein structure of PfSERA5 (Group IV) and Pf-SERA8 (Group I) are shown in Fig. 3a. Pf-sera5 is comprised of four exons and three introns. This exon/intron structure is common in most Group II to IV sera genes, with few exceptions. Alternatively, Group I of Laverania species, which includes 


\section{a}

\section{P. falciparum SERA5 (3D7)}

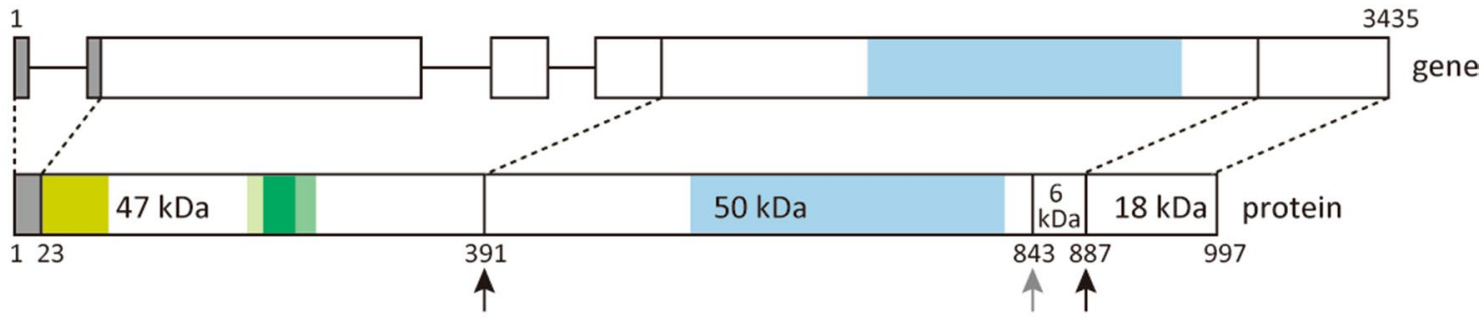

P. falciparum SERA8 (3D7)

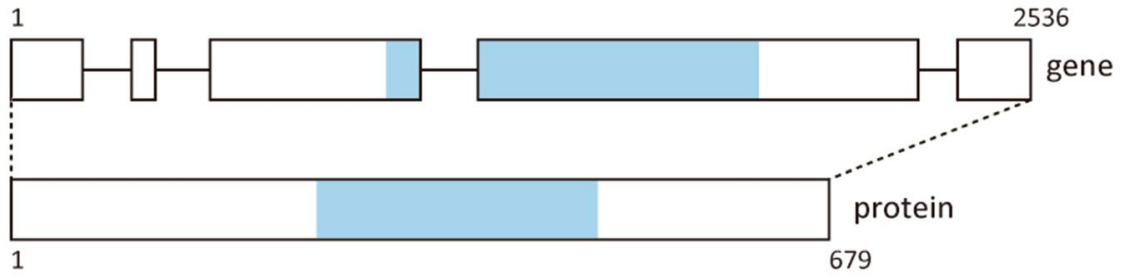
signal peptide
octamer repeat
13 mer insertion/deletion region
serine repeat
17 mer dimorphic region
papain-like domain
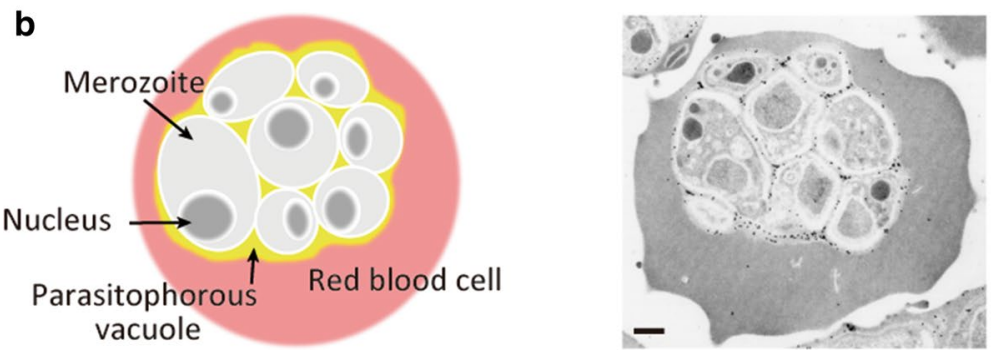

Fig. 3 Structure and localization of SERA. a Schematic representation of the Plasmodium falciparum SERA5 and SERA8 genes and proteins; P. falciparum 3D7 strain was used as a reference for the illustration. The gene IDs in PlasmoDB [18] were PF3D7_0207600 and PF3D7_0207300, for Pf-SERA5 and Pf-SERA8, respectively. Amino acid positions for the Pf-SERA5 fragments are: 47 kDa corresponding to 23-390 aa; 50 kDa: 391-842 aa; 6 kDa: 843-886 aa; and 18 kDa: 887-997 aa. Black arrows indicate the PfSUB1 cleavage sites. The $50 \mathrm{kDa}+6 \mathrm{kDa}$ fragment is further processed by an unknown protease (gray arrow). b Localization of Pf-SERA5 in the parasitophorous vacuole. Left image shows an infected red blood cell with schizont stage malaria parasites inside the parasitophorous vacuole. Right image, N-terminal antisera was used to localize Pf-SERA5 within a schizont parasitized red blood cell. Viewed under JEM-1230 transmission electron microscope (18,000× magnification). SERA5 conjugated gold particles were observed inside the parasitophorous vacuole. Scale-bar: b, $500 \mathrm{~nm}$

Pf-sera8, is comprised of five exons and four introns, while all other Plasmodium Group I sera gene contain six exons and five introns [17]. In terms of protein structure, all Plasmodium SERAs contain a papain-like cysteine protease domain in the middle of the molecule; however, the canonical cysteine center is substituted with a serine in Group IV SERA. Group I SERA retains the conserved cysteine residue in the protease domain, and proteolytic activity was observed in rodent parasites [21]. The possibility of protease activity for Group
IV SERA was dismissed by reverse genetics analysis of Pf-SERA5; as substituting the serine residue for alanine had no phenotypic consequence [22].

The role of SERA was primarily determined using $P$. falciparum and P. berghei. As shown in Fig. 4, SERA functions at several stages of the malaria parasite lifecycle, in both host and mosquito vectors. Knockout/ disruption of nine $P f$-sera genes suggested that $P f$-sera 5 and $P f$-sera 6 are indispensable in the asexual blood stage [23, 24]. In addition, conditional mutagenesis confirmed the necessity of Pf-sera6 [25]. These data 


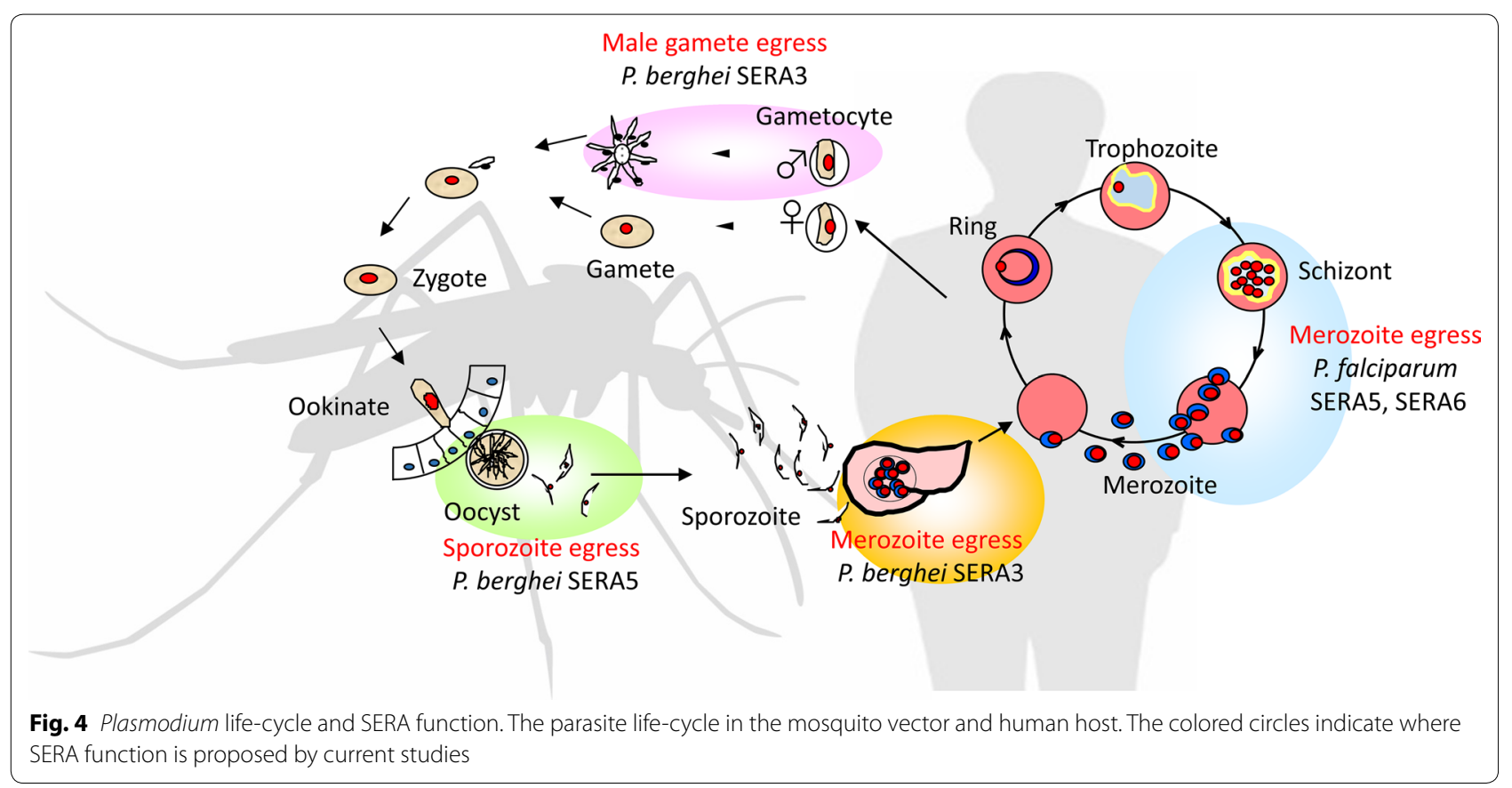

imply that Pf-SERA5 and Pf-SERA6 have crucial roles in malaria blood-stage infection.

\section{Asexual blood stage}

Pf-SERA5 is a blood stage antigen expressed during late trophozoite and schizont stages as a $120 \mathrm{kDa}$ precursor and secreted into the parasitophorous vacuole (PV) after removal of the signal peptide [26] (Fig. 3b). Pf-SERA5 is cleaved by an essential $P$. falciparum subtilisin-like serine protease 1 (PfSUB1) into $47 \mathrm{kDa}, 56 \mathrm{kDa}$ and $18 \mathrm{kDa}$ fragments [27] (Fig. 3a). The $47 \mathrm{kDa}$ fragment is linked to the $18 \mathrm{kDa}$ fragment via a disulfide bond and localizes to the merozoite surface $[28,29]$. The $56 \mathrm{kDa}$ fragment containing the papain-like catalytic domain is further cleaved by an unknown protease to $50 \mathrm{kDa}$ and $6 \mathrm{kDa}$ fragments just before parasite egress $[22,27,28]$. Since the cleavage of the $56 \mathrm{kDa}$ fragment is sensitive to cysteine protease inhibitors, for example E64, leupeptin and iodoacetoamide, the unknown protease is also believed to be a cysteine protease [28]. PfSUB1-mediated Pf-SERA5 processing is required for efficient egress from host erythrocytes [30].

Although protease activity of Pf-SERA5 has been refuted using native [31] and recombinant Pf-SERA5 [22], schizont rupture and merozoite release subsequently occur following Pf-SERA5 processing, and a block in the proteolytic processing of Pf-SERA5 is correlated to a block in erythrocyte rupture [27, 32-34]. Hence, Pf-SERA5 contributes an essential function that cannot be compensated by other members of the
SERA family [22]. Furthermore, conditional disruption of Pf-SERA5 suggested its importance in regulating the kinetics and efficiency of parasite egress [35]. Pf-SERA5 also interacts with calcium dependent protein kinase 1 (PfCDPK1). Phosphorylation of Pf-SERA5 by PfCDPK1 boosts cytosolic $\mathrm{Ca}^{2+}$ levels, which serves as a trigger for merozoite egress [36].

Pf-SERA6, Group III, possesses the canonical cysteine residue in the active site cleft of the papain-like central domain, and is expressed simultaneously with PfSERA5 in the PV. Pf-SERA6 is also cleaved by PfSUB1, transforming Pf-SERA6 into an active cysteine protease [37]. Thomas et al. [38] showed that parasites lacking PfSUB1 fail to rupture the PV membrane (PVM). In contrast, Pf-SERA6-null parasites successfully ruptured the PVM yet failed to rupture RBC membrane to release the merozoites. Thus, Pf-SERA6 activated by PfSUB1 is required for the disassembly of the RBC cytoskeleton. Although, both Pf-SERA5 and Pf-SERA6 have been shown to be essential in parasite egress, the precise role and mechanism of Pf-SERA5 remains unclear.

The conserved nature of SUB1 across all Plasmodium species infers its importance to the parasite. In fact, the consensus sequence of the SUB1 cleavage site, (Val/ Leu/Ile)-Xaa-(Gly/Ala)-Paa, where Xaa represent any amino acid residue and Paa denotes a non-polar residue, except for Leu [27], is well conserved in Group II to IV SERA [17]. SUB1 is also well conserved among Plasmodium species [39]. 


\section{Liver stage}

Plasmodium berghei SUB1 (PbSUB1)-mediated processing activates $P$. berghei SERA3 (Pb-SERA3) [40]. $\mathrm{Pb}-\mathrm{SERA} 3$, the orthologue of Pf-SERA6, is expressed in the late liver stage $[40,41]$ and is believed to function as a cysteine protease. Involvement in PVM rupture was suggested when disruption of the PVM did not occur in the presence of the cysteine protease inhibitor E64 [42]. Under E64-treatment, host hepatocytes and PVM were intact and no Pb-SERA3 secretion to the host cell cytoplasm was observed in P. berghei-infected cells [41]. The proteolytic processing is an essential process of PVM rupture in liver stage parasites [40]. In addition, it was suggested that Pb-SERA3 contributes to host cell death by activating other parasite or host proteins following PVM breakdown [41].

\section{Oocyst and sporozoite stages}

The function of Group I SERA was analyzed using Pb-SERA5, the orthologue of Pf-SERA8. Pb-SERA5 is expressed in oocyst and sporozoite stages [16]. Midgut sporozoite egress from oocysts was prevented by disruption of Pb-SERA5 [21] (ECP1 in Aly et al. [21] is identical to Pb-SERA5). Pb-SERA5 does not have the conserved SUB1 cleavage site and the detailed underlying molecular mechanism remains unknown; however, the sequence of Group I is highly conserved among Plasmodium species. The timing of the expression of Group I SERA is similar between Pb-SERA5 and Pf-SERA8 [18]. Moreover, $\mathrm{Pb}-\mathrm{SERA} 5-\mathrm{knock}$ out parasites affected circumsporozoite protein (CSP) processing, suggesting a possible involvement of Pb-SERA5 in CSP maturation [21]. Altogether, these data suggest that Group I SERA functions in sporozoite egress from oocysts.

\section{Gametocyte stages}

It was also shown that PbSUB1 plays a critical role in male gamete egress [43]. During the process, Pb-SERA3 expressed in male gametocytes is cleaved by PbSUB1 when it is discharged into the PV. This proteolytic process mediates PVM rupture and parasite egress.

\section{Polymorphism of sera genes}

The malaria vaccine candidate antigen Pf-SERA5 has an octamer repeat and serine repeat at the $\mathrm{N}$-terminal region (Fig. 3a). The number of both repeats vary among field isolates as well as laboratory parasite lines [44, 45]. In addition, there are 13-mer insertion/deletion and 17-mer dimorphic regions close to the serine stretch region $[44,45]$. One epitope in the octamer region at amino acid positions 59-72 (PF3D7_0207600) [18] was shown to be the target of parasite growth inhibitory antibodies in vitro $[8,46]$ and was perfectly conserved in 445 worldwide isolates of $P$. falciparum [45]. Polymorphic sites in non-repeat regions of Pf-sera5 (2562 bp) was limited to only 24 nucleotide sites. The ratio of $\mathrm{dN}$ (the number of non-synonymous substitutions per nonsynonymous site), dS (the number of synonymous substitutions per synonymous site), and Tajima's $D$ test did not detect any strong signature for positive selection in the non-repeat regions of Pf-sera5 [45]. The nucleotide diversity of non-repeat regions of Pf-sera 5 is comparable to the housekeeping genes of P-type $\mathrm{Ca}^{2+}$-ATPase (serca) and adenylosuccinate lyase (adsl) (Fig. 5). This is an advantage in the current efforts to develop an effective vaccine, since overcoming antigenic diversity remains a key challenge with most vaccine candidates tested in clinical trials. In contrast to Pf-sera5, major immune target antigen genes of $P$. falciparum, such as apical membrane protein 1 (ama1), csp and merozoite surface antigen 1 ( $m s p 1)$, show high nucleotide diversity $\left(\theta_{\mathrm{s}}\right)$ and significant levels of positive selection $(\mathrm{dN}>\mathrm{dS})$ driven most probably by immune pressure [47] (Fig. 5). Notably, an early stage clinical trial and follow-up study in Uganda for the vaccine candidate based on the Pf-SERA5 $47 \mathrm{kDa}$ domain, showed that vaccination reduced clinical malaria in the vaccine cohort [48], although assessment of strainspecific response is needed.

As shown in Fig. 6, all Pf-sera genes exhibit low diversity/polymorphism rate, similar to Pf-sera5.

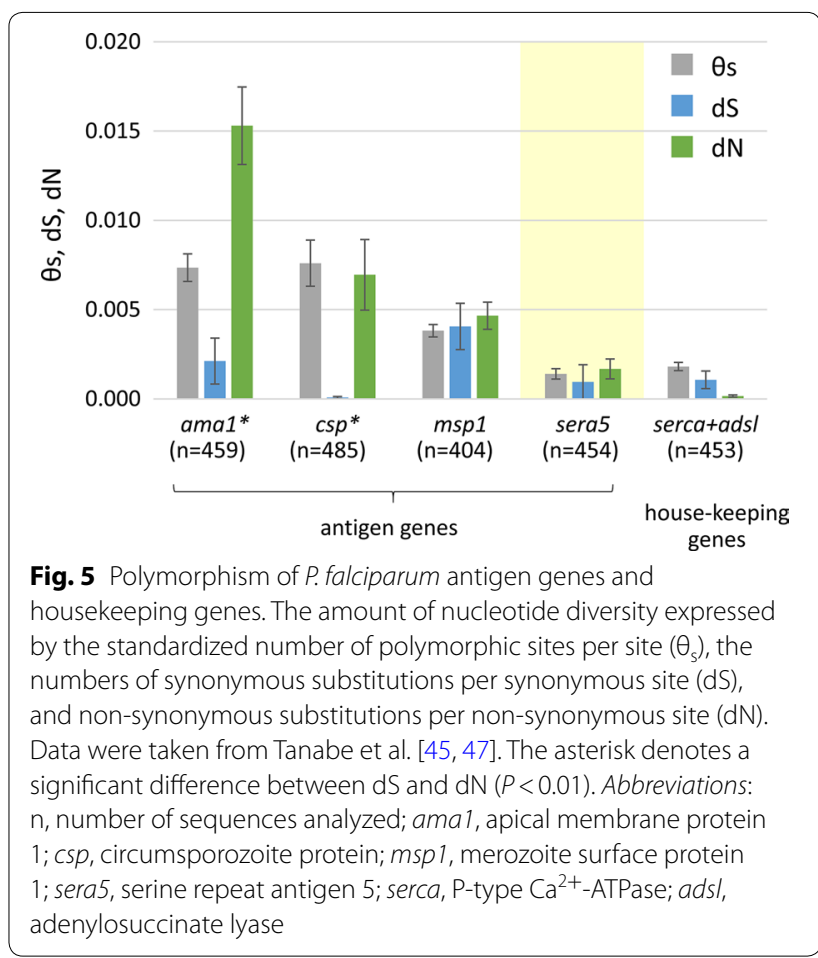




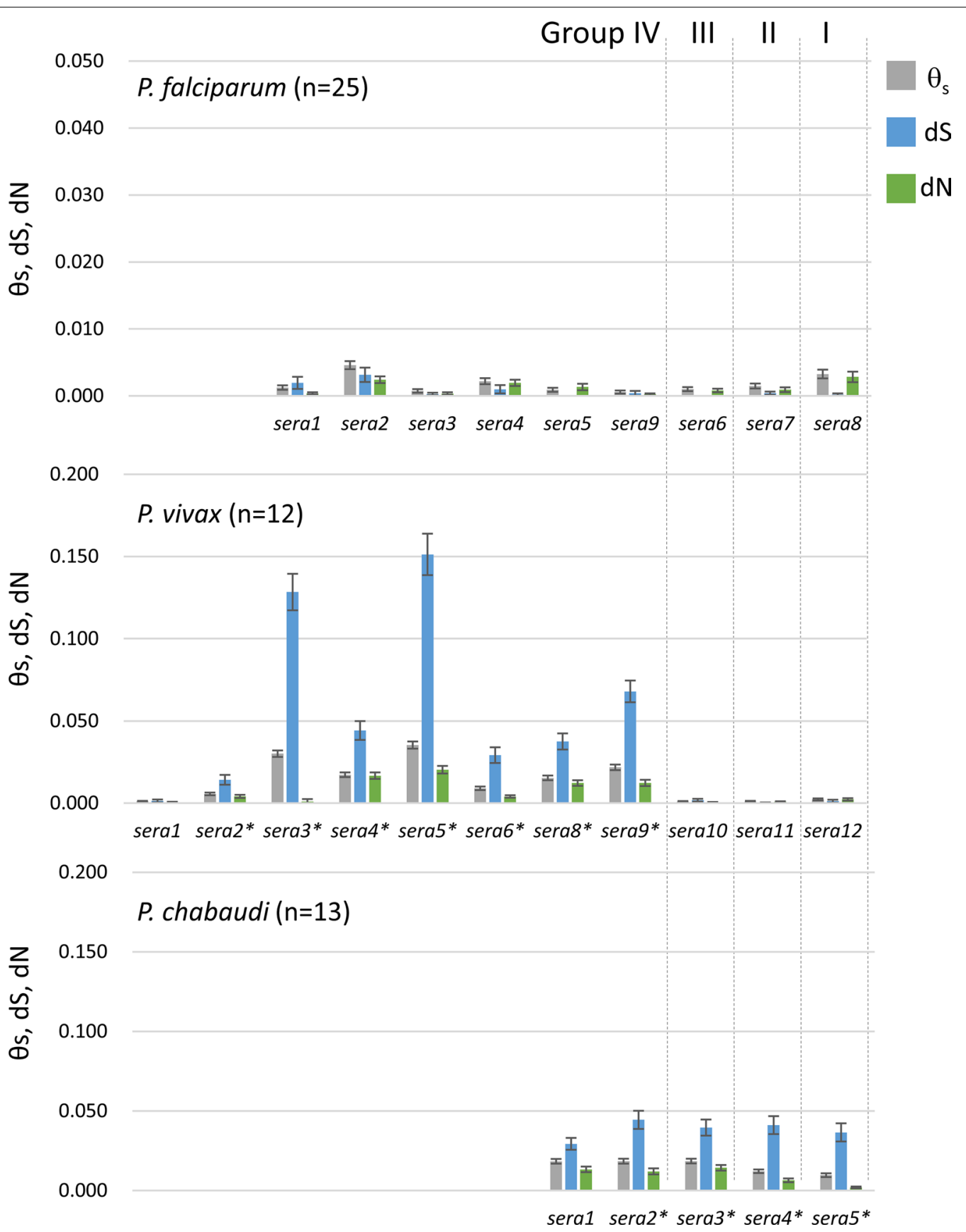

Fig. 6 Sequence diversities $\left(\theta_{s}\right), \mathrm{dS}, \mathrm{dN}$ in $P$. falciparum, P. vivax, and $P$. chabaudi sera genes. The amount of nucleotide diversity as expressed by the standardized number of polymorphic sites per site $\left(\theta_{s}\right)$ were calculated using DnaSP v5.10.01 [61]. The numbers of synonymous substitutions per synonymous site ( $d S$ ) and of nonsynonymous substitutions per nonsynonymous site ( $d N$ ) were calculated using MEGA X [60]. As some strains of $P v$-sera7 contain stop codons in the predicted open reading frame, sequences of $P V$-sera 7 were omitted from analysis. The asterisk denotes significant difference between $\mathrm{dS}$ and $\mathrm{dN}(P<0.01)$

However, sera genes in other Plasmodium species have different polymorphic features. Polymorphisms in Group IV Plasmodium vivax (Pv)-sera genes are much higher than those of Group I to III Pv-sera genes. The nucleotide diversities of the five sera genes of Plasmodium chabaudi $(P c)$ are similar among the gene family members. Pc-sera nucleotide diversity was higher than all $P f$-sera genes and Group I to III $P v$-sera genes; however, lower than certain Group IV Pv-sera genes. No significant positive selection $(\mathrm{dN}>\mathrm{dS})$ was 
detected for both Pv-sera and Pc-sera genes, rather it is evident that purifying selection $(\mathrm{dS}>\mathrm{dN})$ is acting on Pv-sera and Pc-sera genes marked with an asterisk in Fig. 6. The purifying selection is thought to be due to functional and structural constraints. This suggests that sera genes under selective pressure must be functionally active.

The major immune target antigen genes of $P$. falciparum show positive selection [47, 49-52]. In contrast, the polymorphism of $P f$-sera 5 is very limited with no significant immune pressure detected. The $\mathrm{N}$-terminal $47 \mathrm{kDa}$ domain of Pf-SERA5 induced growth inhibitory antibodies in mice $[12,53]$ and humans $[11,12,54]$. Although a large amount of Pf-SERA5 is released into P. falciparum-infected human blood [29], the seroconversion rate of the $47 \mathrm{kDa}$ domain among residents of a malaria endemic area in the Solomon Islands was much lower than that of Pf-MSP1 [14]. These data suggest that the $47 \mathrm{kDa}$ fragment is less immunogenic. A recent study showed that the $47 \mathrm{kDa}$ fragment bound to host vitronectin, which in turn bound to other host proteins to prevent phagocytosis of merozoites, highlighting a camouflage strategy that facilitates the parasite escape from the host immune response [55]. This phenomena was observed in the Ugandan Phase Ib trial, which reported a linear decrease in the number of vaccine responders with increasing age from $6-10$ yearsold, 11-15 years-old, and 16-20 years-old, with only a few vaccine responders observed in the adult cohort (21-35 years-old) [48]. In contrast, $100 \%$ seroconversion was reported in a Phase Ia trial with malaria-naive Japanese adults [14]. These observations suggest that exposure to repeated natural infections in malariaendemic areas, as well as the ability of the $47 \mathrm{kDa}$ fragment to bind to host vitronectin, dampens the immune response, thereby, causing the vaccinated individual to become 'tolerant'. Without immune-driven selection pressure, Pf-SERA5 polymorphisms remain limited.

Differences in the number of Group IV sera genes are notable among primate, rodent, and avian Plasmodium species (Fig. 1b). In P. berghei, simultaneous disruption of the two Group IV sera genes, Pb-sera 1 and Pb-sera2, did not affect parasite growth [56], suggesting nonessential or auxillary roles. Similarly, disruption of Plasmodium yoelii (Py)-sera1 and Py-sera2, which showed higher transcription levels in virulent compared to avirulent parasite lines, did not affect parasite survival in vivo, although the lethality of the parasite was attenuated [57]. In contrast, Pf-SERA5 is essential for $P$. falciparum growth [22, 27, 32-34]. These observations suggest that gene duplication in Group IV sera genes in primate Plasmodium species has occurred for functions required for host-adaptation.

\section{Conclusions}

Of all Plasmodium SERA, Pf-SERA5 is currently the most studied, and was the first member to be identified before publication of the $P$. falciparum genome project. While initial studies showed Pf-SERA5 to be involved in membrane rupture and parasite egress, the cysteine protease active center of this (pseudo)protease is replaced with a serine residue, and several seminal studies have failed to show protease activity. Group IV SERA, to which PfSERA5 belongs, consists of several serine-type SERA resulting from repeated gene duplication events. Hence, Group IV SERA may have acquired new functions during the evolution of the malaria parasite. In P. falciparum, SERA5 was shown to function as a regulator of merozoite egress and was also found to interact with host proteins to evade host immunity; meanwhile, P. yoelii SERA2 was inferred to be associated with parasite virulence. In P. vivax, synonymous mutations found to accumulate in certain Group IV sera genes suggest their contribution to the parasite's life-cycle. The acquisition of various functions through gene duplication would provide high parasite adaptability allowing for the expansion of host range and increased fitness. The cysteine-type SERA (Group III), Pf-SERA6 and Pb-SERA3, showed protease activity and are responsible for merozoite egress. However, the activity of Pb-SERA3 appears multifunctional, as it has been detected in the host liver and is also selectively expressed in the male gametocyte. Further, gene disruption of Pb-sera5 (Group I SERA) demonstrated its role in sporozoite egress from oocysts. Hence, it is clear that all functions of the SERA family have not yet been elucidated and characterized, and thus, requires further investigation. In parasitology, multigene families are described primarily as being associated with immune evasion strategies by amplifying serologically different allelic types. However, the observations in the sera gene family suggest the possibility for other important roles of these families. The development of innovative methods would lead to an increased understanding of the SERA family.

\section{Supplementary information}

Supplementary information accompanies this paper at https://doi. org/10.1186/s13071-020-04044-y.

Additional file 1: Table S1. Accession numbers of Plasmodium sera genes. Gene ID in blue can be found in PlasmoDB (https://plasmodb.org/ plasmo/). Others can be found in the NCBI database (https://www.ncbi. nIm.nih.gov/). Plasmodium sera genes categorized as Group I, II, III, and IV are shaded in pink, yellow, green and blue, respectively.

\section{Abbreviations}

adsl: adenylosuccinate lyase; ama1: apical membrane protein 1; CDPK1: calcium-dependent protein kinase 1; CSP, csp: circumsporozoite protein; MSP1, msp 1: merozoite surface antigen 1; Pb: Plasmodium berghei; Pc: Plasmodium chabaudi; Pf: Plasmodium falciparum; Pg: Plasmodium gallinaceum; Pr: 
Plasmodium relictum; Pv: Plasmodium vivax; PV: parasitophorous vacuole; PVM: parasitophorous vacuole membrane; Py: Plasmodium yoelii; serca: P-type $\mathrm{Ca}^{2+}$-ATPase; SERA, sera: serine repeat antigen; SUB1: subtilisin-like serine protease 1.

\section{Acknowledgements}

Immunoelectron microscope localization of Pf-SERA5 was kindly provided by Professor Motomi Torii (Ehime University, Japan). We would like to thank Editage (www.editage.jp) for English language editing.

\section{Authors' contributions}

NA analyzed sequence data and derived the phylogenetic tree. NA, NMQP, TT and TH contributed in writing the review. All authors read and approved the final manuscript.

\section{Funding}

This work was partially funded by JSPS KAKENHI 25460516 and 17 K08807 grants.

\section{Availability of data and materials Not applicable.}

\section{Ethics approval and consent to participate} Not applicable.

\section{Consent for publication}

Not applicable.

\section{Competing interests}

The authors declare that they have no competing interests.

\section{Author details}

${ }^{1}$ Research Center for Infectious Disease Control, Research Institute for Microbial Diseases, Osaka University, Suita, Osaka 565-0871, Japan. ${ }^{2}$ Department of Malaria Vaccine Development, Research Institute for Microbial Diseases, Osaka University, Suita, Osaka 565-0871, Japan.

Received: 27 December 2019 Accepted: 27 March 2020 Published online: 06 April 2020

\section{References}

1. WHO. World malaria report 2019. Geneva: World Health Organisation; 2019. https://www.who.int/news-room/feature-stories/detail/world -malaria-report-2019. Accessed 19 Dec 2019.

2. Bzik DJ, Li WB, Horii T, Inselburg J. Amino acid sequence of the serine repeat antigen (SERA) of Plasmodium falciparum determined from cloned cDNA. Mol Biochem Parasitol. 1988;30:279-88.

3. Gardner MJ, Tettelin H, Carucci DJ, Cummings LM, Aravind L, Koonin EV, et al. Chromosome 2 sequence of the human malaria parasite Plasmodium falciparum. Science. 1998;282:1126-32.

4. Hall N, Pain A, Berriman M, Churcher C, Harris B, Harris D, et al. Sequence of Plasmodium falciparum chromosomes 1, 3-9 and 13. Nature. 2002:419:527-31.

5. Le Roch KG, Zhou Y, Blair PL, Grainger M, Moch JK, Haynes JD, et al. Discovery of gene function by expression profiling of the malaria parasite life cycle. Science. 2003;301:1503-8.

6. Palacpac NM, Arisue N, Tougan T, Ishii KJ, Horii T. Plasmodium falciparum serine repeat antigen 5 (SE36) as a malaria vaccine candidate. Vaccine. 2011;29:5837-45.

7. Sugiyama T, Suzue K, Okamoto M, Inselburg J, Tai K, Horii T. Production of recombinant SERA proteins of Plasmodium falciparum in Escherichia coli by using synthetic genes. Vaccine. 1996;14:1069-76.

8. Fox BA, Xing-Li P, Suzue K, Horii T, Bzik DJ. Plasmodium falciparum: an epitope within a highly conserved region of the 47-kDa amino-terminal domain of the serine repeat antigen is a target of parasite-inhibitory antibodies. Exp Parasitol. 1997;85:121-34.
9. Pang XL, Horii T. Complement-mediated killing of Plasmodium falciparum erythrocytic schizont with antibodies to the recombinant serine repeat antigen (SERA). Vaccine. 1998;16:1299-305.

10. Pang XL, Mitamura T, Horii T. Antibodies reactive with the N-terminal domain of Plasmodium falciparum serine repeat antigen inhibit cell proliferation by agglutinating merozoites and schizonts. Infect Immun. 1999;67:1821-7.

11. Aoki S, Li J, Itagaki S, Okech BA, Egwang TG, Matsuoka H, et al. Serine repeat antigen (SERA5) is predominantly expressed among the SERA multigene family of Plasmodium falciparum, and the acquired antibody titers correlate with serum inhibition of the parasite growth. J Biol Chem. 2002;277:47533-40.

12. Yagi M, Bang G, Tougan T, Palacpac NM, Arisue N, Aoshi T, et al. Protective epitopes of the Plasmodium falciparum SERA5 malaria vaccine reside in intrinsically unstructured $\mathrm{N}$-terminal repetitive sequences. PLOS ONE. 2014;9:e98460.

13. Okech BA, Nalunkuma A, Okello D, Pang XL, Suzue K, Li J, et al. Natural human immunoglobulin $\mathrm{G}$ subclass responses to Plasmodium falciparum serine repeat antigen in Uganda. Am J Trop Med Hyg. 2001;65:912-7.

14. Horii T, Shirai H, Jie L, Ishii KJ, Palacpac NQ, Tougan T, et al. Evidences of protection against blood-stage infection of Plasmodium falciparum by the novel protein vaccine SE36. Parasitol Int. 2010;59:380-6.

15. Snounou G, Jarra W, Preiser PR. Malaria multigene families: the price of chronicity. Parasitol Today. 2000;16:28-30.

16. Arisue N, Hirai M, Arai M, Matsuoka H, Horii T. Phylogeny and evolution of the SERA multigene family in the genus Plasmodium. J Mol Evol. 2007:65:82-91.

17. Arisue N, Kawai S, Hirai M, Palacpac NM, Jia M, Kaneko A, et al. Clues to evolution of the SERA multigene family in 18 Plasmodium species. PLoS ONE. 2011;6:e17775.

18. Plasmodium genomics resource, PlasmoDB 46. https://plasmodb.org/ plasmo/. Accessed 26 Feb 2020.

19. Honma H, Kawai S, Motooka D, Nakamura S, Tougan T, Horii T, et al. Draft genome sequence of Plasmodium gonderi, a malaria parasite of African Old World monkeys. Genome Announc. 2017;5:e00612-17.

20. Nei M, Rooney AP. Concerted and birth-and death evolution of multigene families. Annu Rev Genet. 2005;39:121-52.

21. Aly AS, Matuschewski K. A malarial cysteine protease is necessary for Plasmodium sporozoite egress from oocysts. J Exp Med. 2005;202:225-30.

22. Stallmach R, Kavishwar M, Withers-Martinez C, Hackett F, Collins CR, Howell SA, et al. Plasmodium falciparum SERA5 plays a non-enzymatic role in the malarial asexual blood-stage lifecycle. Mol Microbiol. 2015;96:368-87.

23. Miller SK, Good RT, Drew DR, Delorenzi M, Sanders PR, Hodder AN, et al. A subset of Plasmodium falciparum SERA genes are expressed and appear to play an important role in the erythrocytic cycle. J Biol Chem. 2002;277:47524-32.

24. McCoubrie JE, Miller SK, Sargeant T, Good RT, Hodder AN, Speed TP, et al. Evidence for a common role for the serine-type Plasmodium falciparum serine repeat antigen proteases: implications for vaccine and drug design. Infect Immun. 2007;75:5565-74.

25. Thomas JA, Collins CR, Das S, Hackett F, Graindorge A, Bell D, et al. Development and application of a simple plaque assay for the human malaria parasite Plasmodium falciparum. PLoS ONE. 2016;11:e0157873.

26. Debrabant A, Maes P, Delplace P, Dubremetz JF, Tartar A, Camus D. Intramolecular mapping of Plasmodium falciparum p126 proteolytic fragments by $\mathrm{N}$-terminal amino acid sequencing. Mol Biochem Parasitol. 1992:53:89-96.

27. Yeoh S, O'Donnell RA, Koussis K, Dluzewski AR, Ansell KH, Osborne SA, et al. Subcellular discharge of a serine protease mediates release of invasive malaria parasites from host erythrocytes. Cell. 2007;131:1072-83.

28. Li J, Matsuoka H, Mitamura T, Horii T. Characterization of proteases involved in the processing of Plasmodium falciparum serine repeat antigen (SERA). Mol Biochem Parasitol. 2002;120:177-86.

29. Boyle MJ, Langer C, Chan JA, Hodder AN, Coppel RL, Anders RF, et al. Sequential processing of merozoite surface proteins during and after erythrocyte invasion by Plasmodium falciparum. Infect Immun. 2014;82:924-36.

30. Agarwal S, Singh MK, Garg S, Chitnis CE, Singh S. Ca(2+)-mediated exocytosis of subtilisin-like protease 1: a key step in egress of Plasmodium falciparum merozoites. Cell Microbiol. 2013;15:910-21. 
31. Sato D, Li J, Mitamura T, Horii T. Plasmodium falciparum serine-repeat antigen (SERA) forms a homodimer through disulfide bond. Parasitol Int 2005:54:261-5.

32. Arastu-Kapur S, Ponder EL, Fonović UP, Yeoh S, Yuan F, Fonović M, et al. Identification of proteases that regulate erythrocyte rupture by the malaria parasite Plasmodium falciparum. Nat Chem Biol. 2008;4:203-13.

33. Blackman MJ. Malarial proteases and host cell egress: an 'emerging' cascade. Cell Microbiol. 2008;10:1925-34.

34. Fairlie WD, Spurck TP, McCoubrie JE, Gilson PR, Miller SK, McFadden GI, et al. Inhibition of malaria parasite development by a cyclic peptide that targets the vital parasite protein SERA5. Infect Immun. 2008;76:4332-44.

35. Collins CR, Hackett F, Atid J, Tan MSY, Blackman MJ. The Plasmodium falciparum pseudoprotease SERA5 regulates the kinetics and efficiency of malaria parasite egress from host erythrocytes. PLoS Pathog 2017;13:e1006453.

36. Iyer GR, Singh S, Kaur I, Agarwal S, Siddiqui MA, Bansal A, et al. Calciumdependent phosphorylation of Plasmodium falciparum serine repeat antigen 5 triggers merozoite egress. J Biol Chem. 2018;293:9736-46.

37. Ruecker A, Shea M, Hackett F, Suarez C, Hirst EM, Milutinovic K, et al. Proteolytic activation of the essential parasitophorous vacuole cysteine protease SERA6 accompanies malaria parasite egress from its host erythrocyte. J Biol Chem. 2012;287:37949-63.

38. Thomas JA, Tan MSY, Bisson C, Borg A, Umrekar TR, Hackett F, et al. A protease cascade regulates release of the human malaria parasite Plasmodium falciparum from host red blood cells. Nat Microbiol. 2018;3:523.

39. Brogi S, Giovani S, Brindisi M, Gemma S, Novellino E, Campiani G, et al. In silico study of subtilisin-like protease 1 (SUB1) from different Plasmodium species in complex with peptidyl-difluorostatones and characterization of potent pan-SUB1 inhibitors. J Mol Graph Model. 2016;64:121-30.

40. Tawk L, Lacroix C, Gueirard P, Kent R, Gorgette O, Thiberge S, et al. A key role for Plasmodium subtilisin-like SUB1 protease in egress of malaria parasites from host hepatocytes. J Biol Chem. 2013;288:33336-46.

41. Schmidt-Christensen A, Sturm A, Horstmann S, Heussler VT. Expression and processing of Plasmodium berghei SERA3 during liver stages. Cell Microbiol. 2008;10:1723-34

42. Sturm A, Amino R, van de Sand C, Regen T, Retzlaff S, Rennenberg A, et al. Manipulation of host hepatocytes by the malaria parasite for delivery into liver sinusoids. Science. 2006:313:1287-90.

43. Pace T, Grasso F, Camarda G, Suarez C, Blackman MJ, Ponzi M, et al. The Plasmodium berghei serine protease PbSUB1 plays an important role in male gamete egress. Cell Microbiol. 2019;21:e13028.

44. Morimatsu K, Morikawa T, Tanabe K, Bzik DJ, Horii T. Sequence diversity in the amino-terminal $47 \mathrm{kDa}$ fragment of the Plasmodium falciparum serine repeat antigen. Mol Biochem Parasitol. 1997;86:249-54.

45. Tanabe K, Arisue N, Palacpac NM, Yagi M, Tougan T, Honma H, et al. Geographic differentiation of polymorphism in the Plasmodium falciparum malaria vaccine candidate gene SERA5. Vaccine. 2012;30:1583-93.

46. Fox BA, Horii T, Bzik DJ. Plasmodium falciparum: fine-mapping of an epitope of the serine repeat antigen that is a target of parasite-inhibitory antibodies. Exp Parasitol. 2002;101:69-72.

47. Tanabe K, Mita T, Palacpac NM, Arisue N, Tougan T, Kawai S, et al. Withinpopulation genetic diversity of Plasmodium falciparum vaccine candidate antigens reveals geographic distance from a Central sub-Saharan African origin. Vaccine. 2013;31:1334-9.

48. Palacpac NM, Ntege E, Yeka A, Balikagala B, Suzuki N, Shirai H, et al. Phase $1 \mathrm{~b}$ randomized trial and follow-up study in Uganda of the blood-stage malaria vaccine candidate BK-SE36. PLoS ONE. 2013;8:e64073.

49. Hughes AL. Circumsporozoite protein genes of malaria parasites (Plasmodium spp.): evidence for positive selection on immunogenic regions. Genetics. 1991;127:345-53.

50. Hughes AL. Positive selection and interallelic recombination at the merozoite surface antigen-I (MSA-1) locus of Plasmodium falciparum. Mol Biol Evol. 1992;9:381-93.

51. Escalante AA, Lal AA, Ayala FJ. Genetic polymorphism and natural selection in the malaria parasite Plasmodium falciparum. Genetics. 1998;149:189-202.

52. Polley SD, Conway DJ. Strong diversifying selection on domains of the Plasmodium falciparum apical membrane antigen 1 gene. Genetics. 2001;158:1505-12.

53. Soe S, Singh S, Camus D, Horii T, Druilhe P. Plasmodium falciparum serine repeat protein, a new target of monocyte dependent antibody-mediated parasite killing. Infect Immun. 2002;70:7182-4.

54. Yagi M, Palacpac NM, Ito K, Oishi Y, Itagaki S, Balikagala B, et al. Antibody titres and boosting after natural malaria infection in BK-SE36 vaccine responders during a follow-up study in Uganda. Sci Rep. 2016;6:34363.

55. Tougan T, Edula JR, Takashima E, Morita M, Shinohara M, Shinohara A, et al. Molecular camouflage of Plasmodium falciparum merozoites by binding of host vitronectin to P47 fragment of SERA5. Sci Rep. 2018;8:5052.

56. Putrianti ED, Schmidt-Christensen A, Arnold I, Heussler VT, Matuschewski $\mathrm{K}$, Silvie $\mathrm{O}$. The Plasmodium serine-type SERA proteases display distinct expression patterns and non-essential in vivo roles during life cycle progression of the malaria parasite. Cell Microbiol. 2010;12:725-39.

57. Huang X, Liew K, Natalang O, Siau A, Zhang N, Preiser PR. The role of serine-type serine repeat antigen in Plasmodium yoelii blood stage development. PLOS ONE. 2013;8:e60723.

58. Katoh K, Standley DM. MAFFT multiple sequence alignment software version 7: improvements in performance and usability. Mol Biol Evol. 2013;30:772-80.

59. Gouy M, Guindon S, Gascuel O. SeaView version 4: a multiplatform graphical user interface for sequence alignment and phylogenetic tree building. Mol Biol Evol. 2010;27:221-4.

60. Kumar S, Stecher G, Li M, Knyaz C, Tamura K. MEGA X: Molecular Evolutionary Genetics Analysis across computing platforms. Mol Biol Evol. 2018;35:1547-9.

61. Librado P, Rozas J. DnaSP v5: a software for comprehensive analysis of DNA polymorphism data. Bioinformatics. 2009;25:1451-2.

\section{Publisher's Note}

Springer Nature remains neutral with regard to jurisdictional claims in published maps and institutional affiliations.

\footnotetext{
Ready to submit your research? Choose BMC and benefit from:

- fast, convenient online submission

- thorough peer review by experienced researchers in your field

- rapid publication on acceptance

- support for research data, including large and complex data types

- gold Open Access which fosters wider collaboration and increased citations

- maximum visibility for your research: over $100 \mathrm{M}$ website views per year
}

At BMC, research is always in progress.

Learn more biomedcentral.com/submissions 